\title{
Molecular Origins of the Zeta Potential
}

\author{
Milan Předota, ${ }^{* \dagger}$ Michael L. Machesky ${ }^{\ddagger}$, David J. Wesolowski ${ }^{\S}$ \\ ${ }^{\dagger}$ Institute of Physics and Biophysics, Faculty of Science, University of South Bohemia, Branisovska 1760, \\ 370 o5 Ceske Budejovice, Czech Republic \\ ${ }^{\ddagger}$ University of Illinois, Illinois State Water Survey, 2204 Griffith Drive, Champaign, IL 61820-7495 \\ ${ }^{\S}$ Chemical Sciences Division, Oak Ridge National Laboratory, Oak Ridge, TN 37831-6110
}

\section{Supporting Information}

\section{SIMULATED SYSTEMS}

Movies of electroosmotic flows are available here: http://home.prf.jcu.cz/ predota/zeta/si-movies/
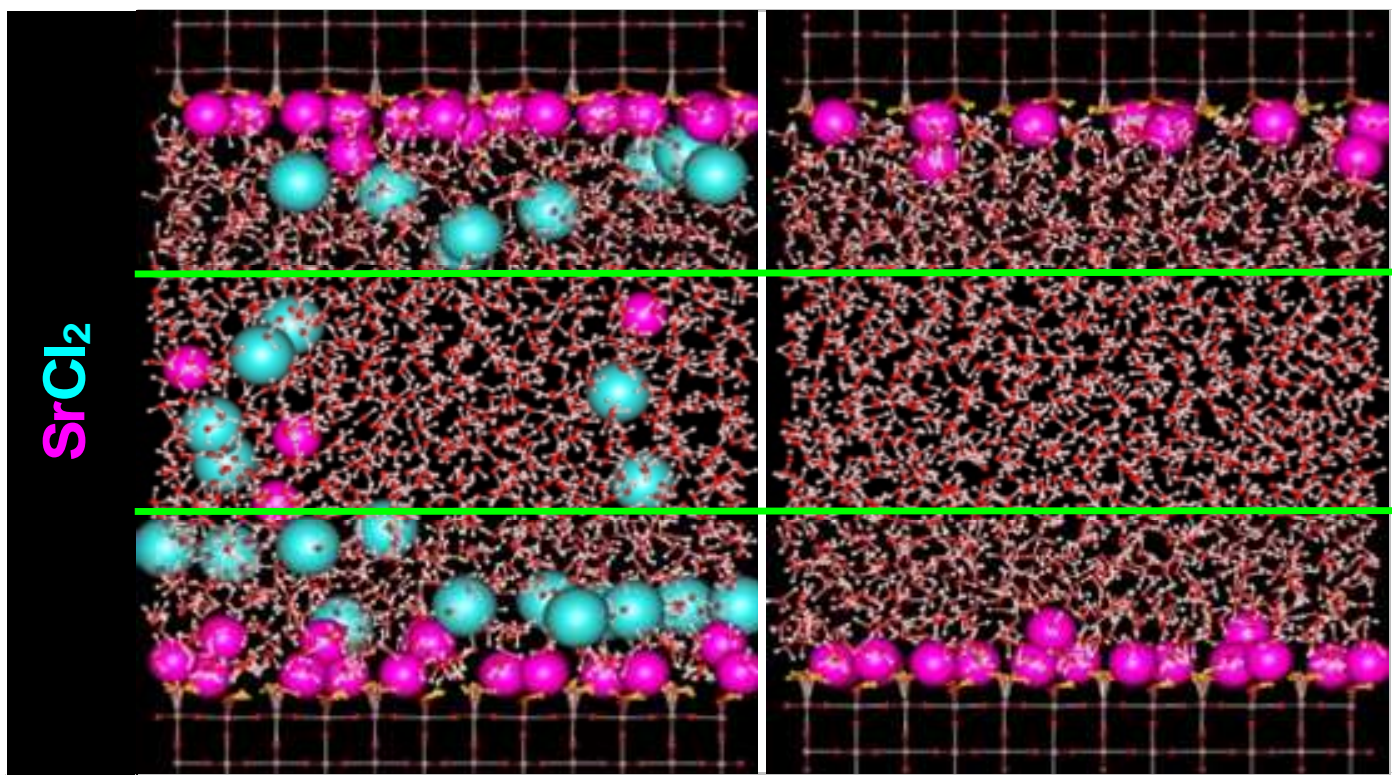

Figure SI1. Snapshots of simulated systems for $\mathrm{SrCl}_{2}$ and surface charge density $-0.4 \mathrm{C} / \mathrm{m}^{2}$. Left panel is for bulk concentration about $0.24 \mathrm{M}$, right for system with only cations. The green lines at the distance about $15 \AA$ from the rutile (110) surface indicate the approximate boundary between the interfacial and bulk regions. 


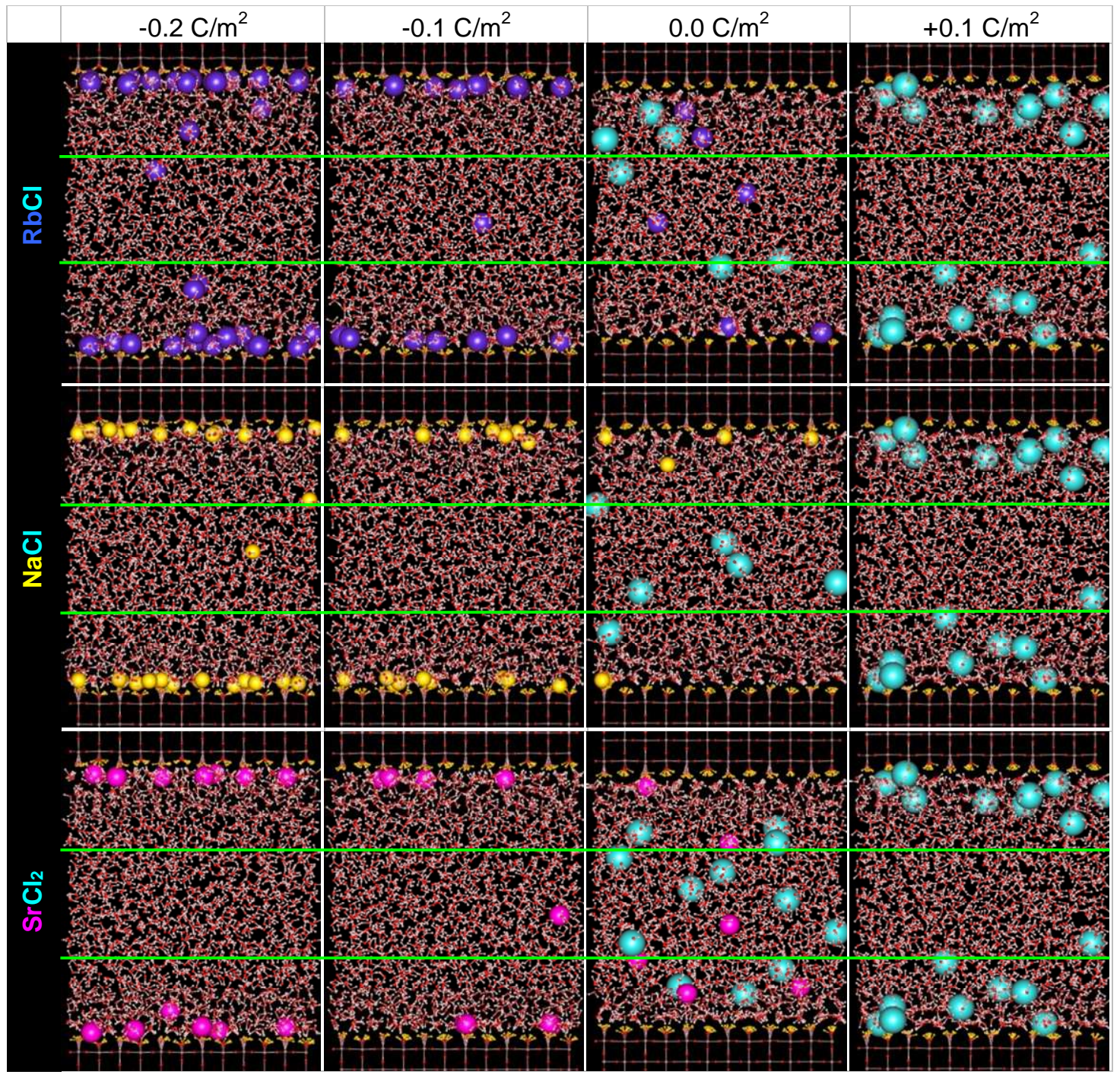

Figure SI2. Snapshots of simulated systems with only counter-ions, i.e., $\mathrm{Rb}^{+}, \mathrm{Na}^{+}$, and $\mathrm{Sr}^{2+}$ for -0.2 and $-0.1 \mathrm{C} / \mathrm{m}^{2}$, and $\mathrm{Cl}^{-}$ for $+0.1 \mathrm{C} / \mathrm{m}^{2}$ (the last column represents one simulation but is included in each row to complete the series). For the neutral surfaces, a limited number of ion pairs $\left(6 \mathrm{RbCl}\right.$ or $\mathrm{NaCl}$ or $\left.\mathrm{SrCl}_{2}\right)$ were added to observe the behavior of ions at low concentrations. The green lines at the distance about $15 \AA$ from the rutile (110) surface indicate the approximate boundary between the interfacial and bulk regions.

Simulations where the total amount of ions was adjusted to keep concentrations in the bulk region approximately constant for a given cation and all surface charge conditions are identified in bold blue in Tables SI1-SI3.

These simulations indicated by red italics in Tables $\mathrm{S}_{1}-\mathrm{S}_{3}$ and labeled in graphs as o $\mathrm{M}$ represent simulations at the lowest concentrations, i.e., systems containing only counter-ions to compensate surface charge and no coions (charged surfaces) or a very small number of cations and anions in the case of neutral surfaces. For the neutral surface, the limit of zero bulk concentrations leads to a trivial system of pure water with exactly zero electroosmotic mobility and ZP. The simulation data for neutral (uncharged) surfaces and low ion concentrations are represented by simulations with 6 cations. 
For the monovalent cations, $\mathrm{Rb}^{+}$and $\mathrm{Na}^{+}$, bulk concentrations were calculated as the average of the timeaveraged concentrations of cations and anions. For simulations with $\mathrm{Sr}^{2+}$ the average density of $\mathrm{SrCl}_{2} \mathrm{was} \mathrm{used}$, i.e., the average of $\mathrm{Sr}^{2+}$ and half the $\mathrm{Cl}^{-}$density.

Table SI1. Number of ions in the simulations of $\mathrm{RbCl}$ solutions, bulk concentrations, electroosmotic mobility $\mu_{\mathrm{H}_{2} \mathrm{O}}$ determined from NEMD simulations and calculated ZPs $(\zeta)^{\mathrm{a}}$.

\begin{tabular}{|c|c|c|c|c|c|c|}
\hline $\begin{array}{l}\text { surface charge } \\
\text { density }\left[\mathrm{C} / \mathrm{m}^{2}\right]\end{array}$ & $\mathrm{pH}$ & $\#$ of $\mathrm{Rb}^{+}$ & $\#$ of $\mathrm{Cl}^{-}$ & $\begin{array}{l}\text { bulk conc. } \\
\text { [M] }\end{array}$ & $\begin{array}{c}\mu_{\mathrm{H}_{2} \mathrm{O}} \\
{\left[10^{-8} \mathrm{~m}^{2} /(\mathrm{V} . \mathrm{s})\right]}\end{array}$ & $\zeta[\mathrm{mV}]$ \\
\hline \multirow{5}{*}{-0.2} & \multirow{5}{*}{9.4} & 36 & o & 0.05 & 2.36 & -30.0 \\
\hline & & 38 & 2 & 0.12 & 1.93 & -24.5 \\
\hline & & 48 & 12 & 0.40 & 0.75 & -9.6 \\
\hline & & 55 & 19 & 0.61 & 0.33 & $-4 \cdot 3$ \\
\hline & & 72 & 36 & 1.06 & 0.23 & -3.0 \\
\hline \multirow{4}{*}{-0.1} & \multirow{4}{*}{$7 \cdot 4$} & 18 & o & 0.05 & 2.47 & -31.5 \\
\hline & & 30 & 12 & 0.39 & 0.97 & -12.3 \\
\hline & & 36 & 18 & 0.55 & -0.09 & 1.1 \\
\hline & & 54 & 36 & 1.02 & -0.60 & $7 \cdot 7$ \\
\hline \multirow{3}{*}{0.0} & \multirow{3}{*}{$5 \cdot 4$} & 6 & 6 & 0.17 & -0.66 & 8.4 \\
\hline & & 18 & 18 & 0.51 & -1.36 & $17 \cdot 3$ \\
\hline & & 36 & 36 & 0.99 & -0.94 & 12.0 \\
\hline \multirow{4}{*}{+0.1} & \multirow{4}{*}{$2.8^{\mathrm{b}}$} & o & 18 & 0.08 & -4.70 & 59.9 \\
\hline & & 12 & 30 & 0.47 & -3.03 & 38.5 \\
\hline & & 18 & 36 & 0.66 & -2.52 & 32.1 \\
\hline & & 27 & 45 & 0.95 & -1.89 & 24.1 \\
\hline
\end{tabular}

${ }^{\mathrm{a}}$ The external field was $2.1 \times 10^{8} \mathrm{~V} / \mathrm{m}$. ${ }^{\mathrm{b}}$ Estimated $\mathrm{pH}$ value. 
Table SI2. As for Table S1, but for $\mathrm{NaCl}$ solutions.

\begin{tabular}{|c|c|c|c|c|c|c|}
\hline $\begin{array}{l}\text { surface charge } \\
\text { density }\left[\mathrm{C} / \mathrm{m}^{2}\right]\end{array}$ & $\mathrm{pH}$ & $\#$ of $\mathrm{Na}^{+}$ & \# of $\mathrm{Cl}^{-}$ & $\begin{array}{l}\text { bulk conc. } \\
{[\mathrm{M}]}\end{array}$ & $\begin{array}{c}\mu_{\mathrm{H}_{2} \mathrm{O}} \\
{\left[10^{-8} \mathrm{~m}^{2} /(\mathrm{V} . \mathrm{s})\right]}\end{array}$ & $\zeta[\mathrm{mV}]$ \\
\hline \multirow{6}{*}{-0.2} & \multirow{6}{*}{8.9} & 36 & o & 0.04 & 1.34 & -17.1 \\
\hline & & 38 & 2 & 0.07 & 0.20 & -2.5 \\
\hline & & 42 & 6 & 0.16 & -0.64 & 8.2 \\
\hline & & 48 & 12 & 0.29 & -1.15 & 14.7 \\
\hline & & 51 & 15 & 0.39 & -1.66 & 21.1 \\
\hline & & 72 & 36 & 0.92 & -1.37 & 17.4 \\
\hline \multirow{7}{*}{-0.1} & \multirow{7}{*}{$7 \cdot 4$} & 18 & o & 0.04 & 1.47 & -18.8 \\
\hline & & 20 & 2 & 0.08 & 0.52 & -6.6 \\
\hline & & 24 & 6 & 0.14 & -0.21 & 2.7 \\
\hline & & 27 & 9 & 0.21 & -0.33 & 4.2 \\
\hline & & 30 & 12 & 0.33 & -1.03 & 13.0 \\
\hline & & 36 & 18 & 0.43 & -1.62 & 20.6 \\
\hline & & 54 & 36 & 0.87 & -1.53 & 19.5 \\
\hline \multirow{4}{*}{0.0} & \multirow{4}{*}{$5 \cdot 4$} & 6 & 6 & 0.13 & -1.72 & 21.8 \\
\hline & & 12 & 12 & 0.23 & -2.16 & 27.5 \\
\hline & & 18 & 18 & 0.41 & -1.90 & 24.1 \\
\hline & & 36 & 36 & 0.78 & -2.04 & 26.0 \\
\hline \multirow{6}{*}{+0.1} & \multirow{6}{*}{$2.8^{\mathrm{b}}$} & o & 18 & 0.08 & -4.70 & 59.9 \\
\hline & & 2 & 20 & 0.14 & -4.29 & 54.6 \\
\hline & & 6 & 24 & 0.27 & $-3 \cdot 5^{2}$ & 44.8 \\
\hline & & 12 & 30 & 0.40 & -2.89 & 36.7 \\
\hline & & 18 & 36 & 0.61 & -2.70 & 34.4 \\
\hline & & 30 & 48 & 0.91 & -2.08 & 26.5 \\
\hline
\end{tabular}


Table $\mathrm{SI}_{3}$. As Table $\mathrm{S}_{1}$, but for $\mathrm{SrCl}_{2}$ solutions.

\begin{tabular}{|c|c|c|c|c|c|c|}
\hline $\begin{array}{l}\text { surface charge } \\
\text { density }\left[\mathrm{C} / \mathrm{m}^{2}\right]\end{array}$ & $\mathrm{pH}$ & $\#$ of $\mathrm{Sr}^{2+}$ & $\#$ of $\mathrm{Cl}^{-}$ & $\begin{array}{l}\text { bulk conc. } \\
{[\mathrm{M}]}\end{array}$ & $\begin{array}{c}\mu_{\mathrm{H}_{2} \mathrm{O}} \\
{\left[10^{-8} \mathrm{~m}^{2} /(\mathrm{V} . \mathrm{s})\right]}\end{array}$ & $\zeta[\mathrm{mV}]$ \\
\hline \multirow{4}{*}{-0.4} & \multirow{4}{*}{$7 \cdot 3^{\mathrm{a}}$} & 36 & o & 0.00 & 0.03 & -0.3 \\
\hline & & 42 & 12 & 0.09 & -2.90 & 36.9 \\
\hline & & 49 & 26 & 0.24 & -2.85 & 36.3 \\
\hline & & 54 & 36 & 0.41 & -1.64 & 20.9 \\
\hline \multirow{5}{*}{-0.2} & \multirow{5}{*}{$5 \cdot 9$} & 18 & o & 0.00 & 0.30 & $-3 \cdot 9$ \\
\hline & & 21 & 6 & 0.07 & -1.24 & 15.8 \\
\hline & & 27 & 18 & 0.15 & -2.97 & 37.8 \\
\hline & & 31 & 26 & 0.29 & -1.80 & 23.0 \\
\hline & & 36 & 36 & 0.44 & -1.44 & 18.3 \\
\hline \multirow{5}{*}{-0.1} & \multirow{5}{*}{$5 \cdot 3$} & 9 & o & 0.01 & 1.03 & -13.1 \\
\hline & & 12 & 6 & 0.05 & -1.36 & $17 \cdot 3$ \\
\hline & & 18 & 18 & 0.20 & -1.69 & 21.5 \\
\hline & & 22 & 26 & 0.33 & -1.73 & 22.0 \\
\hline & & 27 & 36 & 0.49 & -1.41 & 17.9 \\
\hline \multirow{3}{*}{0.0} & \multirow{3}{*}{4.5} & 6 & 12 & 0.21 & -0.28 & 3.6 \\
\hline & & 10 & 20 & 0.26 & -1.72 & 21.9 \\
\hline & & 16 & 32 & 0.41 & -1.30 & 16.6 \\
\hline \multirow{3}{*}{+0.1} & \multirow{3}{*}{$2.8^{\mathrm{b}}$} & o & 18 & 0.08 & -4.70 & 59.9 \\
\hline & & 7 & 32 & 0.30 & -2.32 & 29.6 \\
\hline & & 9 & 36 & 0.40 & -2.12 & 27.0 \\
\hline
\end{tabular}




\section{DISTANCE-DEPENDENT MOBILITIES OF IONS}

The electroosmotic mobility of water, which is our main interest, is averaged in $0.125 \AA$ wide bins parallel the surface directly as $\mu_{\mathrm{H}_{2} \mathrm{O}}(z)=\left\langle v_{\mathrm{H}_{2} \mathrm{O}}^{x}(z)\right\rangle / E_{x}$. For ions, the velocity of water is subtracted to obtain the mobility of ions relative to water, $\mu_{\text {ion }}=\left[\left\langle v_{\text {ion }}^{x}(z)\right\rangle-\left\langle v_{\mathrm{H}_{2} \mathrm{O}}^{x}(z)\right\rangle\right] / E_{x}$. The mobilities of water and ions as a function of distance from the immobile surface for a series of simulations resulting in about $0.44 \mathrm{M}$ bulk concentration of $\mathrm{RbCl}$ are plotted in Figure $\mathrm{SI}_{3}$, for $0.41 \mathrm{M} \mathrm{NaCl}$ in Figure $\mathrm{SI}_{4}$, and for about $0.28 \mathrm{M} \mathrm{SrCl}_{2}$ in Figure $\mathrm{SI}_{5}$.
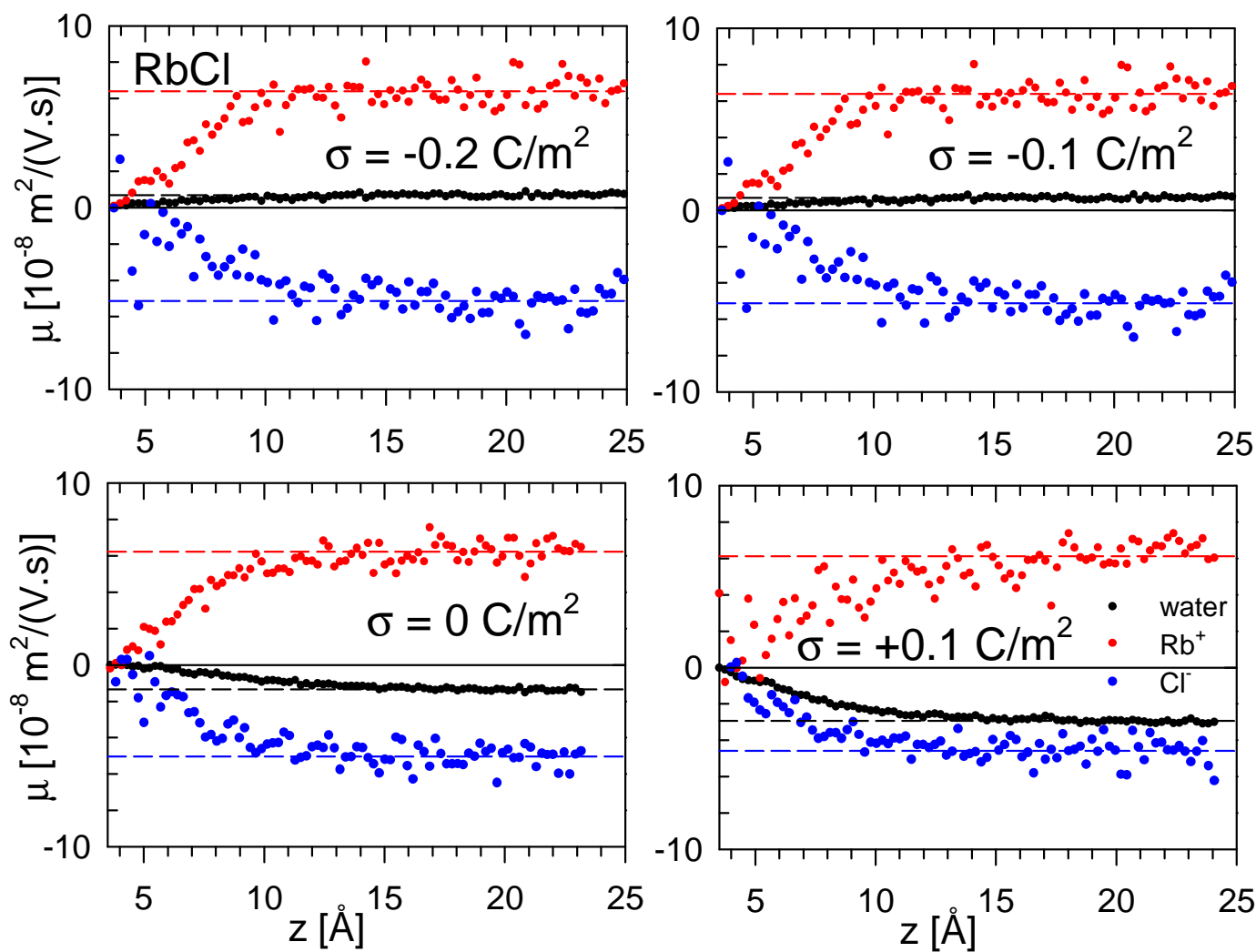

Figure SI3. Mobilities of water and ions $\left(\mathrm{Rb}^{+}, \mathrm{Cl}^{-}\right)$with respect to distance above the rutile surface as determined from NEMD simulations. The bulk concentration of $\mathrm{RbCl}$ is about $0.44 \mathrm{M}$. The horizontal dashed lines indicate the averaged bulk mobilities over $z>15$ Å. 

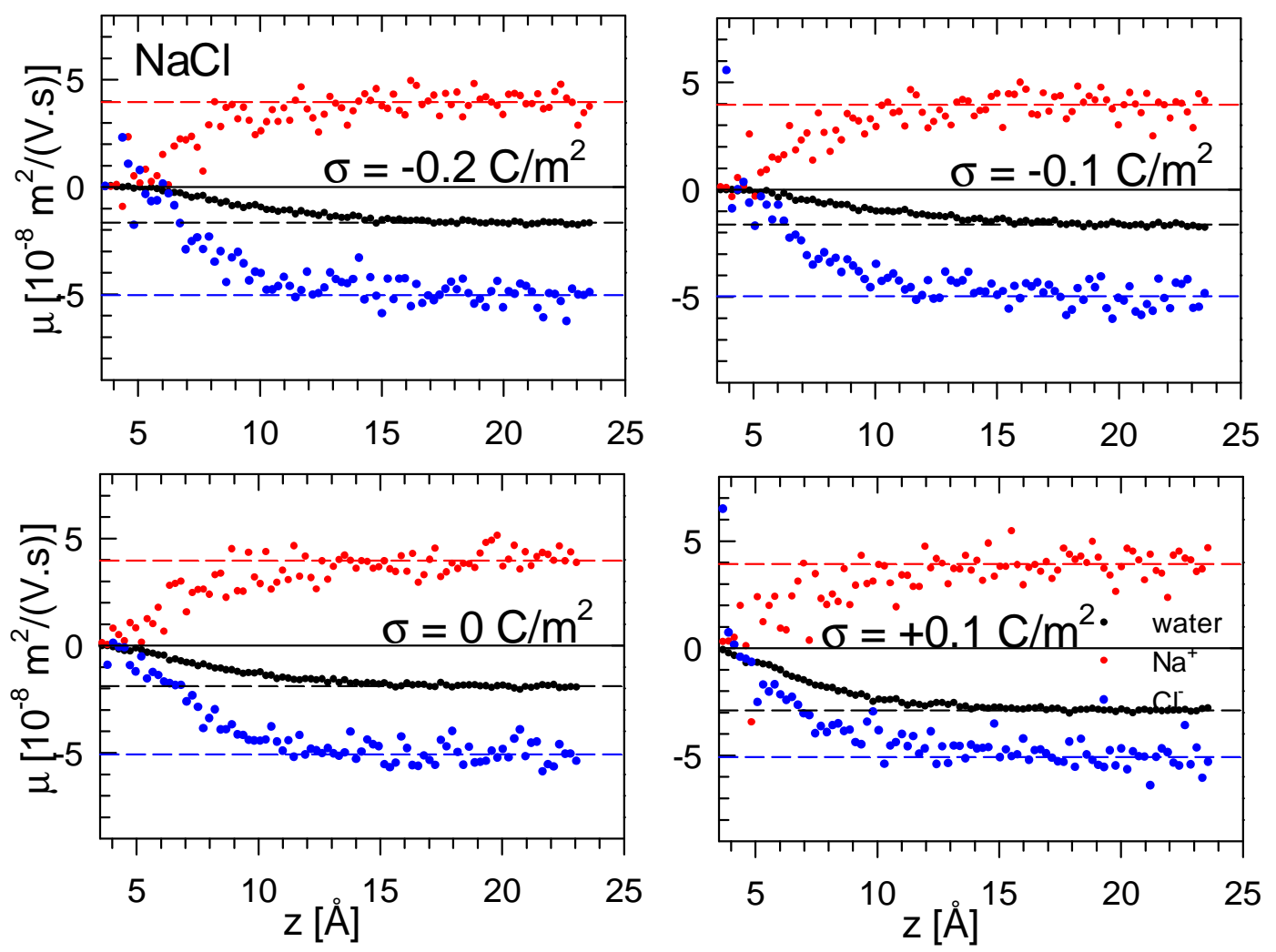

Figure SI4. Mobilities of water and ions ( $\mathrm{Na}+, \mathrm{Cl}-)$ with respect to distance above the rutile (110) surface as determined from NEMD. The bulk concentration of $\mathrm{NaCl}$ is about $0.41 \mathrm{M}$. The horizontal dashed lines indicate the averaged bulk mobilities. 

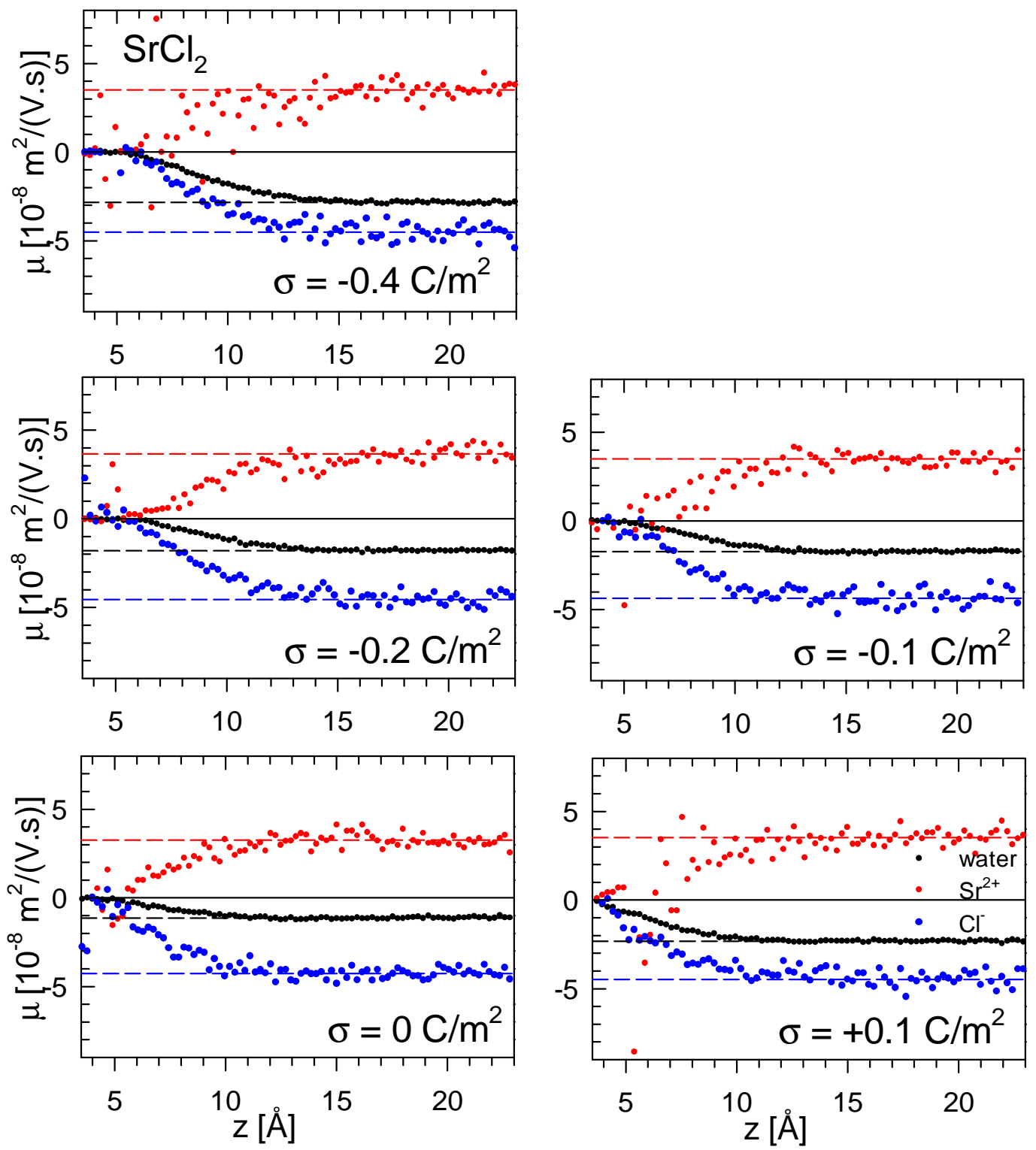

Figure SI5. Mobilities of water and ions $\left(\mathrm{Sr}^{2+}, \mathrm{Cl}^{-}\right)$with respect to distance above the rutile (110) surface as determined from NEMD. The bulk concentration of $\mathrm{SrCl}_{2}$ is about $0.28 \mathrm{M}$. The horizontal dashed lines indicate the averaged bulk mobilities.

In all cases the mobilities are nearly zero very close to the surface and then increase further from the surface. ${ }^{1}$ This increase is nearly linear up to about $10 \AA$ and then a plateau is reached at about $12-15 \AA$ from the surface, where bulk values are attained. The bulk values of ionic mobilities are independent of the surface and equal the values one would obtain from surface-free bulk simulations of water and ions in an external electric field. The simulated bulk ionic mobilities depend linearly on the bulk concentration, $\mu_{\text {ion }}^{\text {sim }}=\mu_{\text {ion }}^{\text {sim } 0}+k c$; the mobilities $\mu_{\text {ion }}^{\text {sim, } 0}$ extrapolated to zero concentration and the slope $k$ are given in Table $\mathrm{SI}_{4}$ together with experimental values. The simulated mobilities extrapolated to zero concentrations reach about $92 \%\left(\mathrm{Rb}^{+}\right), 93 \%\left(\mathrm{Na}^{+}\right), 78 \%$ 
$\left(\mathrm{Sr}^{2+}\right)$ and $69-76 \%\left(\mathrm{Cl}^{-}\right)$of the experimental mobilities calculated as $\mu=\Lambda_{\mathrm{m}} /\left(e z N_{A}\right)$ from the tabulated molar conductivities ${ }^{2} \Lambda_{\mathrm{m}}$, where $\mathrm{z}$ is the charge of ion in units of elementary charge $e$. The simulated mobilities of $\mathrm{Cl}^{-}$ decrease with increasing interaction with the cations. The agreement between simulated and experimental mobilities is very good for $\mathrm{Rb}^{+}$and $\mathrm{Na}^{+}$, but less favorable for $\mathrm{Sr}^{2+}$ and $\mathrm{Cl}^{-}$. This discrepancy is a deficit of the potential models of water and ions used, ${ }^{3,4}$ which were not fitted to mobility data, but only to energetic data and structure. Given this shortcoming, the performance of the potential models is good. On the other hand, this discrepancy limits the accuracy of our electroosmotic results, as the motion of water is induced by dragging forces between ions and water, which are intimately related to the ionic mobilities. Similar discrepancies were already observed in simulations of ionic mobilities in homogeneous solutions of SPC/E water. ${ }^{5}$

Table SI4. Simulated mobilities of ions extrapolated to zero concentration $\mu_{\text {ion }}^{\operatorname{sim} 0}$ and the corresponding slope $k$, experimental mobilities at zero concentration $\mu_{\text {ion }}^{\exp }$ and molar conductivities $\Lambda_{\mathrm{m}}$.

\begin{tabular}{|c|c|c|c|c|}
\hline ion & $\begin{array}{c}\mu_{\mathrm{ion}}^{\operatorname{sim}, 0} \\
{\left[10^{-8} \mathrm{~m}^{2} /(\mathrm{V} . \mathrm{s})\right]}\end{array}$ & $\begin{array}{c}k \\
{\left[10^{-8} \mathrm{~m}^{2} /(\mathrm{V} . \mathrm{s} . \mathrm{mol})\right]}\end{array}$ & $\begin{array}{c}\mu_{\text {ion }}^{\exp } \\
{\left[10^{-8} \mathrm{~m}^{2} /(\mathrm{V} . \mathrm{s})\right]^{a}}\end{array}$ & $\begin{array}{c}\Lambda_{\mathrm{m}} \\
{\left[10^{-4} \mathrm{~m}^{2} \cdot \mathrm{S} \cdot \mathrm{mol}^{-1}\right]^{b}}\end{array}$ \\
\hline $\mathrm{Rb}^{+}$ & 7.4 & -2.2 & 8.06 & 77.8 \\
\hline $\mathrm{Na}^{+}$ & 4.8 & -2.0 & 5.19 & 50.08 \\
\hline $\mathrm{Sr}^{2+}$ & 4.8 & -4.1 & 6.16 & $59.4^{c}$ \\
\hline $\mathrm{Cl}^{-}$ & $\begin{array}{l}-6.0^{d} \\
-5.7^{e} \\
-5.4^{f}\end{array}$ & $\begin{array}{l}2.0 \\
1.7 \\
2.9\end{array}$ & $\begin{array}{l}-7.91 \\
-7.91 \\
-7.91\end{array}$ & $\begin{array}{l}76.31 \\
76.31 \\
76.31\end{array}$ \\
\hline
\end{tabular}

${ }^{a}$ Calculated from $\Lambda_{\mathrm{m}} ;{ }^{b}$ From reference ${ }^{2} ;{ }^{c}$ Value of $\Lambda_{\mathrm{m}}\left(1 / 2 \mathrm{Sr}^{2+}\right) ;{ }^{d} \mathrm{RbCl},{ }^{e} \mathrm{NaCl},{ }^{f} \mathrm{SrCl}_{2}$ solutions.

\section{RELATION BETWEEN STREAMING VELOCITY OF FLUID AND STREAMING VELOCITY OF WATER}

Simple, but perhaps not intuitive calculations show that generally the resulting velocity of water $v_{\mathrm{H}_{2} \mathrm{O}}$ within a layer is not exactly equal to the center-of-mass velocity of that layer ( $v_{\mathrm{COM}}$, which is identified as the velocity of the fluid layer)

$$
\vec{v}_{\mathrm{COM}}-\vec{v}_{\mathrm{H}_{2} \mathrm{O}}=\vec{E} \frac{m_{+} \mu_{+}+m_{-} \mu_{-}}{m_{+}+m_{-}+m_{\mathrm{H}_{2} \mathrm{O}}},
$$

where $m_{+}, m_{-}$, and $m_{\mathrm{H}_{2} \mathrm{O}}$ are the overall masses of all cations, anions and water molecules in the layer and the mobilities of cations and anions, $\mu_{+}$and $\mu_{-}$, are positive and negative, respectively. However, the difference between $v_{\mathrm{H}_{2} \mathrm{O}}$ and $v_{\mathrm{COM}}$ is proportional to the ratio of the total masses of ions and water molecules in a layer, which is, depending on atomic masses of ions, about $1 \%$ for a $0.3 \mathrm{M}$ ionic solution (in about 55.6 $\mathrm{M}$ water). Moreover, the terms in the numerator tend to cancel when the products $m_{+} \mu_{+}$and $m_{-} \mu_{-}$are of comparable magnitudes, but of opposite sign - as is our case. Therefore, for practical purposes, we do not need to discriminate between the terms 'electroosmotic mobility of water' $\left(v_{\mathrm{H}_{2} \mathrm{O}}\right)$ and 'electroosmotic mobility of fluid' $\left(v_{\mathrm{COM}}\right)$. However, note that in the interfacial layers the difference between $v_{\mathrm{COM}}$ and $v_{\mathrm{H}_{2} \mathrm{O}}$ can be more significant since the local concentrations of ions reach tens of molal even for low bulk ion concentrations ${ }^{1}$, while the concentrations of oppositely charged ions of can be negligible in the same layer. Still, this difference is not important for the determination of ZP, which is determined exclusively by the relative motion of fluid in the bulk with respect to the surface. And, in the bulk, the difference between $v_{\mathrm{COM}}$ and $v_{\mathrm{H}_{2} \mathrm{O}}$ is negligible. 


\section{ELECTROSTATIC POTENTIALS}

The profiles of the electrostatic potentials (calculated by double integration of the charge distribution as described in Delgado et al. $^{6}$ ) are shown in Figure SI6. When all contributions from surface atoms, water molecules (calculated from atomistic distributions of oxygen and hydrogen atoms) and ions are considered (Figure SI6, left panel), the electrostatic potential is dominated by damped oscillations around zero, originating from the water contributions due to preferred orientation of water dipoles and inhomogeneous water density. As expected, at neutral and positively charged surfaces, the profiles do not depend on the choice of cations. The effect of cations increases with negative surface charge, but is limited mostly to the region of inner-sphere and first outer-sphere adsorption peaks, i.e., around 3.5-8 $\AA$. When the contribution from water molecules is not included, as would be the case in continuum theories of the solid-liquid interface, which do not incorporate the effect of water orientation and differences in positions of oxygens and hydrogens, we obtain the very different and much smoother profiles of electrostatic potential shown in Figure SI6, right panel. None of these profiles indicate any specific position to equate with ZP values. Moreover, the scale of observed electrostatic potentials is significantly larger (of the order of volts at distances of 3-4 $\AA$ where the mobile layers begin) compared to typical values of $\mathrm{ZP}$ (of the order of tens of $\mathrm{mV}$ ). The potential arising from surface charge and ions (right panel) shows a trend observed also in Figure 4 of Předota et al., ${ }^{1}$ i.e., that strongly adsorbing sodium and strontium cations overcompensate the negative surface charge, resulting in regions of positive electrostatic potential at these surfaces, a feature not occurring with $\mathrm{Rb}^{+}$. This phenomenon has been observed before in simulations of $\mathrm{NaCl}$ aqueous solutions in contact with negatively charged silica surfaces ${ }^{7}$ and other works mentioned in the main text. The big contrast between the profiles with and without contributions from water molecules (note also the different scales of both panels) stresses the key role of water structure and water orientation on interfacial electrostatics. Particularly, the preferential and surface-induced orientation of water molecules can effectively significantly weaken the effect of surface charge, as documented by the similarity of the electrostatic profiles in the left panel of Figure SI6 as opposed to large differences in the profiles in the right panel of Figure SI6.

The analysis and discussion of electrostatic profiles, together with our previous analysis of the dynamical properties of the interface ${ }^{8}$ lead to a conclusion that it is not possible to locate a clearly-defined slipping plane, that is a specific point on the profiles of electrostatic properties, that can be linked to the macroscopicallyobservable ZP. The concept of ZP as an electrostatic potential at a slip plane separating the mobile and immobile regions is conceptually useful for the basic notion of electrokinetic phenomena and interaction of particles, but in the light of molecular data is evidently too oversimplified. The determination of the shear plane (or providing facts against this over-simplified concept) was one of the targets of our publications, where we studied the distance-dependence of the diffusivity of water ${ }^{8,9}$ and viscosity., ${ }^{9,10}$

Our non-equilibrium molecular dynamics results demonstrate that the whole interfacial region contributes to the value of ZP and that the electrostatics and dynamics both play key roles. 

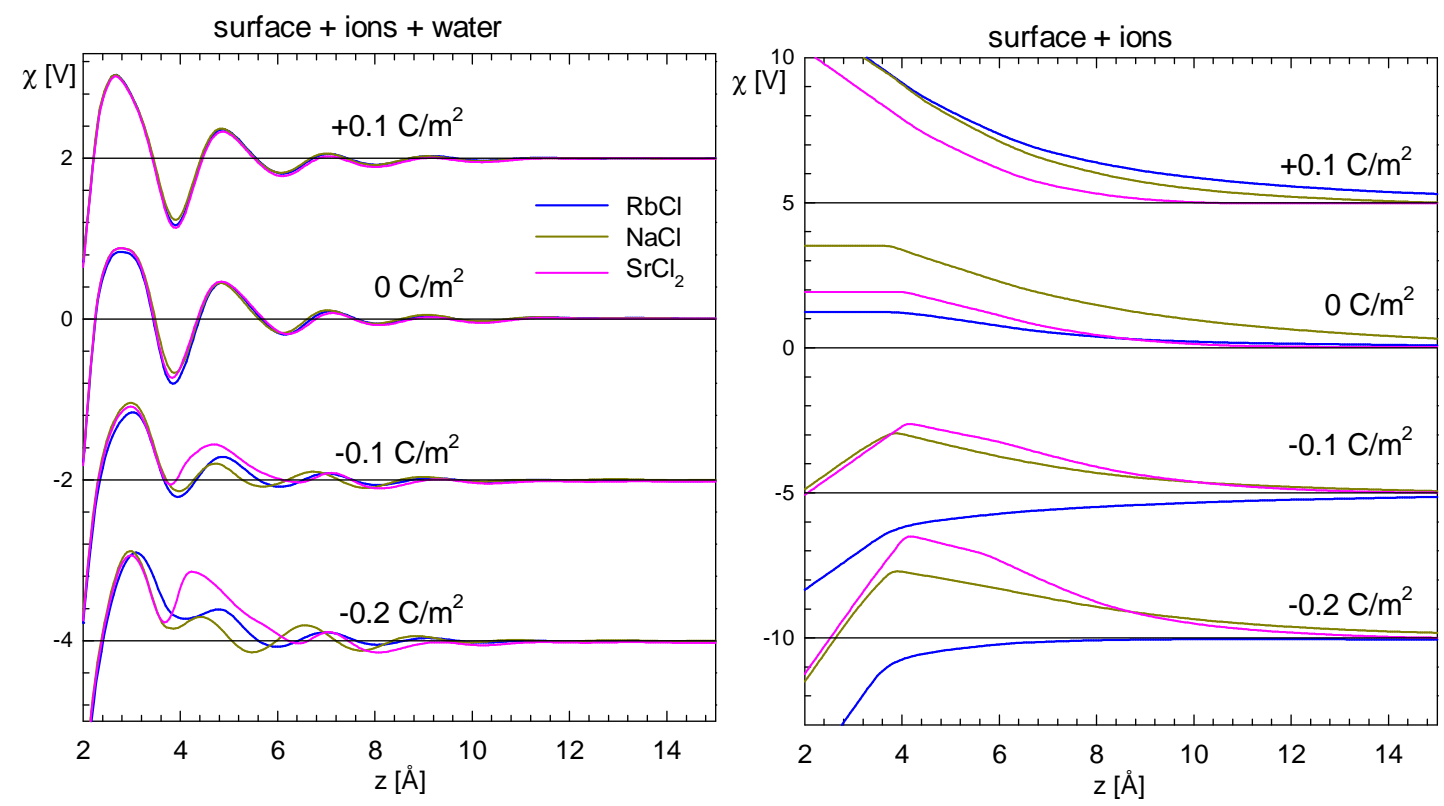

Figure SI6. Electrostatic potential at the rutile/aqueous solution interface calculated from all contributions (surface + water + ions, left) and without contribution from water molecules (surface + ions, right). All curves were shifted to zero potential in the bulk; the offset by multiples of 2, resp. 5, is artificial to distinguish the curves for differently charged surfaces. The systems identified in bold in Tables SI1-SI3 are considered.

\section{APPROXIMATE PREDICTIONS OF ZP FROM THEORY COMBINED WITH EQUILIBRIUM MOLECULAR DYNAMICS}

In principle, one can calculate electroosmotic streaming velocities from a combination of two known axial distributions for each studied system. The first is the axial profile of charge density at the interface, $\rho_{q}(z)$, which is easy to obtain from equilibrium molecular dynamics (EMD) simulations. The second is the profile of viscosity, $\eta(z)$, which is however much more difficult to determine and which we therefore approximate by the profile for pure water at neutral surface, making the otherwise exact theoretical predictions approximate.The offdiagonal component of the pressure tensor at distance $z$ from the surface is calculated as

$$
P_{x z}(z)=E_{x} \int_{z}^{L_{z} / 2} \rho_{q}\left(z^{\prime}\right) d z^{\prime}
$$

where $\rho_{q}(z)$ denotes volume charge density from ions at distance $z$, and $L / 2$ the center of the slab. Note that it is a non-local property, integrating all the drag generated at distances $z^{\prime}$ to the center of slab. A layer at distance $z$ must carry this integrated dragging force towards the surface to keep the system in a steady state. Compared to the pressure tensor profile for a homogenous external force used in our determination of distance-dependent viscosity ${ }^{2}$, which increases from the center of slab towards the surface, the pressure tensor profile under electroosmotic flow is more complex, as the dragging force can be both positive and negative depending on the local volume charge density.

The local shear is given by

$$
\gamma(z)=\frac{P_{x z}(z)}{\eta(z)}
$$

where $\eta(z)$ is the local shear viscosity. From eq. (2) the streaming electroosmotic velocity is calculated as, $v(z)=\int_{0}^{z} \gamma\left(z^{\prime}\right) d z^{\prime}$, resulting in mobility $\mu(z)=\frac{1}{E_{x}} \int_{0}^{z} \frac{P_{x z}\left(z^{\prime}\right)}{\eta\left(z^{\prime}\right)} d z^{\prime}$ independent of $E_{x}$. Note that the 
information on the distance-dependence of the viscosity is required for reasonable prediction of electrokinetic mobility using eq. (2), whereas information on the distance-dependence of permittivity is not needed, since it is implicitly incorporated into the ionic density profiles affecting the pressure tensor via eq. (1).

Using a constant (bulk) value of viscosity is inappropriate due to its sharp increase very near the surface ${ }^{2-3}$, as demonstrated below. On the other hand, determining $\eta(z)$ for each particular surface charge and concentration of ions is a task of comparable difficulty (both in terms of complexity of the simulation and simulation times) to the current NEMD prediction of electroosmotic mobilities. It is in fact even more difficult, because the determination of distance-dependent viscosity requires averaging of streaming velocities and determining the local shear from numerical derivatives or fitting the velocity profile. ${ }^{3,5}$ Determining $\eta(z)$ for each investigated system and substituting into eq. (2) would thus be of little practical importance for determination of ZP, being more demanding than our non-equilibrium simulations of electroosmosis. Therefore, we decided to supply into eq. (2) the profile of $\eta(z)$ determined for pure water in contact with a neutral surface', (see Figure SI7) ignoring its variations with surface charge and presence of ions. The great advantage of this approximation is that we only need a single NEMD simulation for determining $\eta(z)$ and then we can predict ZPs using equilibrium MD simulations yielding the density profiles of ions for a given surface charge density and concentration of ionic species of a given kind. We have a number of equilibrium results from our recent work ${ }^{1}$ and we also ran equilibrium simulations for any new NEMD simulations to be able to validate that the equilibrium and nonequilibrium distributions of ions perpendicular to the surface are statistically equal. This means that this approximate approach yields predictions of ZP at no extra cost. Obviously, with increasing surface charge densities and concentrations of ions the actual viscosity profiles will eventually become significantly different from the one determined for pure water at the neutral surface and the approximation will be less accurate.

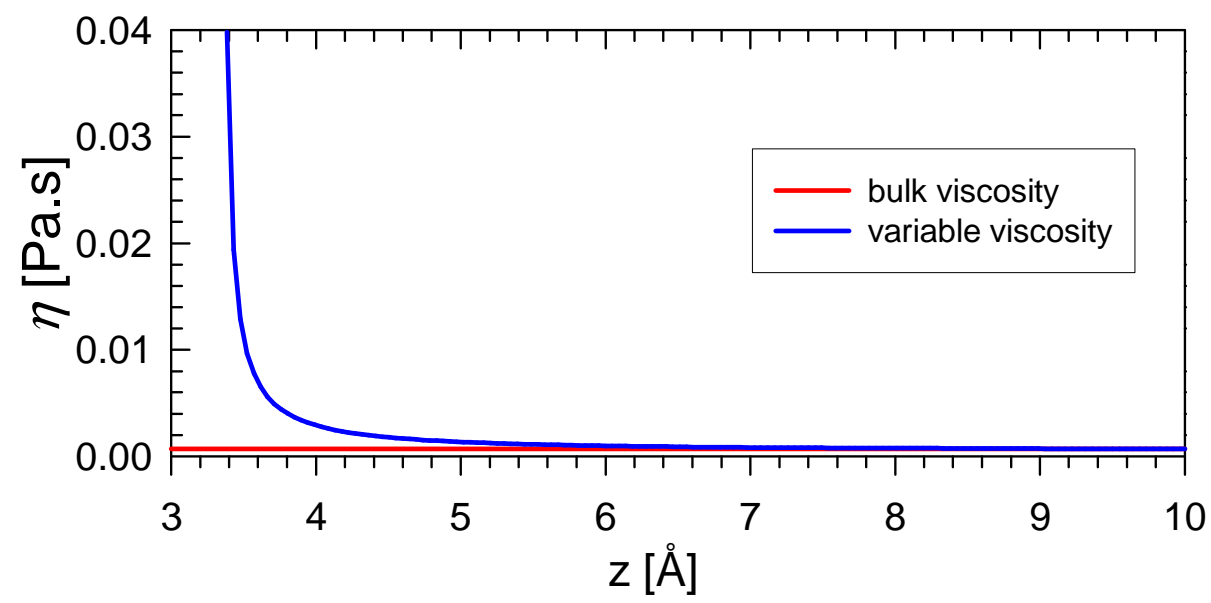

Figure SI7: Inhomogeneous viscosity profile used in our approximate theoretical prediction of ZP from EMD simulations. Smoothed simulation data from Ref. [2].

The approximate EMD predictions, shown in Figures SI8 and SI9 complement the NEMD results presented in Figures 2 and 3 and reveal that the theory works well. However, the quality deteriorates with increasing strength of interactions of ions with the rutile surface. For $\mathrm{RbCl}$, the EMD predictions are nearly quantitative, nicely following the profiles of mobilities as a function of distance from the surface and reaching a plateau in the bulk, from which the ZP is calculated using the Helmholtz-Smoluchowski equation. For $\mathrm{NaCl}$, the approximate prediction is very inaccurate for neutral surface, but for all surfaces the predictions qualitatively agree with the NEMD results and yield negative ZPs for all surfaces. For $\mathrm{SrCl}_{2}$ solutions the EMD predictions are generally very inaccurate. Good agreement is obtained for the positive surface, but for -0.2 and $-0.4 \mathrm{C} / \mathrm{m}^{2}$ surfaces the approximation evidently breaks down due to the strong effect of adsorbed ions on the viscosity profile. Similar trends can be observed in Figure SI9.

The purpose of this section was to present the equations connecting electrostatics with dynamics (viscosity). Equation (2) explains why the contribution of many strongly adsorbed cations to the ZP can be outweighed by a 
minority of mobile anions. In the region of strongly adsorbed cations, the local viscosity is several times larger than in bulk, reducing the local shear and buildup of the ZP. In Figure SIo we demonstrate that ignoring the distance-dependence of viscosity leads to very poor or unacceptable theoretical predictions of electroosmotic mobilities. The solid lines are identical to those in Figure SI8 and were obtained from distance-dependent viscosity profiles, which diverge at distances around 3.4 $\AA$. The short dashed lines correspond to the situation where the stagnant layer is assumed up to $3.4 \AA$, but the bulk viscosity $\left(8.9 \times 10^{-4} \mathrm{~Pa}\right.$.s $)$ is used for larger distances. This curve for $\mathrm{RbCl}$ significantly deviates from the curve with distance-dependent viscosity for the $-0.2 \mathrm{C} / \mathrm{m}^{2}$ surface, particularly close to the surface, where the viscosity deviates significantly from the bulk-value. For $\mathrm{NaCl}$ the curve for step-wise viscosity features a positive mobility at distances around 4-6 $\AA$ due to strong adsorption of $\mathrm{Na}^{+}$at these distances ${ }^{1}$, where however the NEMD simulations show no mobility due to very high viscosity. Due to this incorrect offset, this theoretical prediction coincidentally matches the NEMD data at larger distances very well. Ignoring the presence of a stagnant layer and using the bulk viscosity for all distances (long dashed lines) leads to an unacceptable prediction of mobility for the $-0.2 \mathrm{C} / \mathrm{m}^{2}$ surface. Note that in accord with eq. (1) the pressure tensor $P_{x z}$ for charged surfaces is constant and non-zero close to the surface, with a value given by the integral of the charge of ions in the system up to the center of the slab, which is opposite to that of surface charge, therefore generating constant shear at small distances (see eq. (2)) if constant viscosity is assumed. For the neutral surface, the pressure tensor $P_{x z}$ at small distances is zero, and therefore the shear is zero as well. As a result, the curves for constant and step-wise viscosity for neutral surfaces in Figure Slo overlap.

The approximation of supplying the viscosity profile of pure water instead of the actual viscosity profile for each system gives in fact decent agreement for weakly adsorbing ions and might be used with success even for weakly adsorbing molecules. However, the NEMD approach detailed in the main text provides accurate results of ZPs within the limits of accuracy of the molecular models and sampling of configurations. 

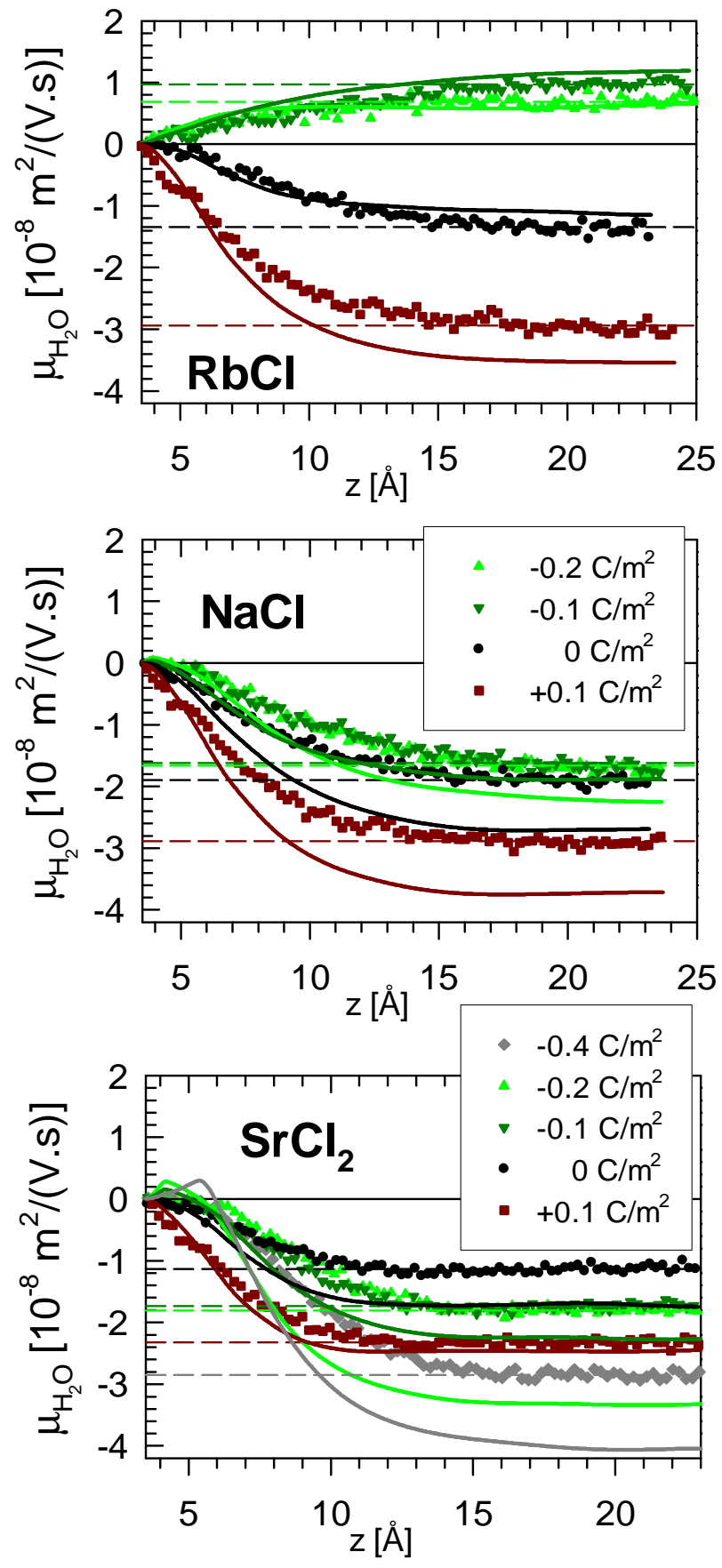

Figure SI8: Electroosmotic mobilities of water in $0.44 \mathrm{M} \mathrm{RbCl}$ (top), $0.41 \mathrm{M} \mathrm{NaCl}$ (middle), and $0.31 \mathrm{SrCl}_{2}$ solutions from NEMD (points) and EMD (lines). The horizontal dashed lines indicate the averaged NEMD bulk mobilities. 

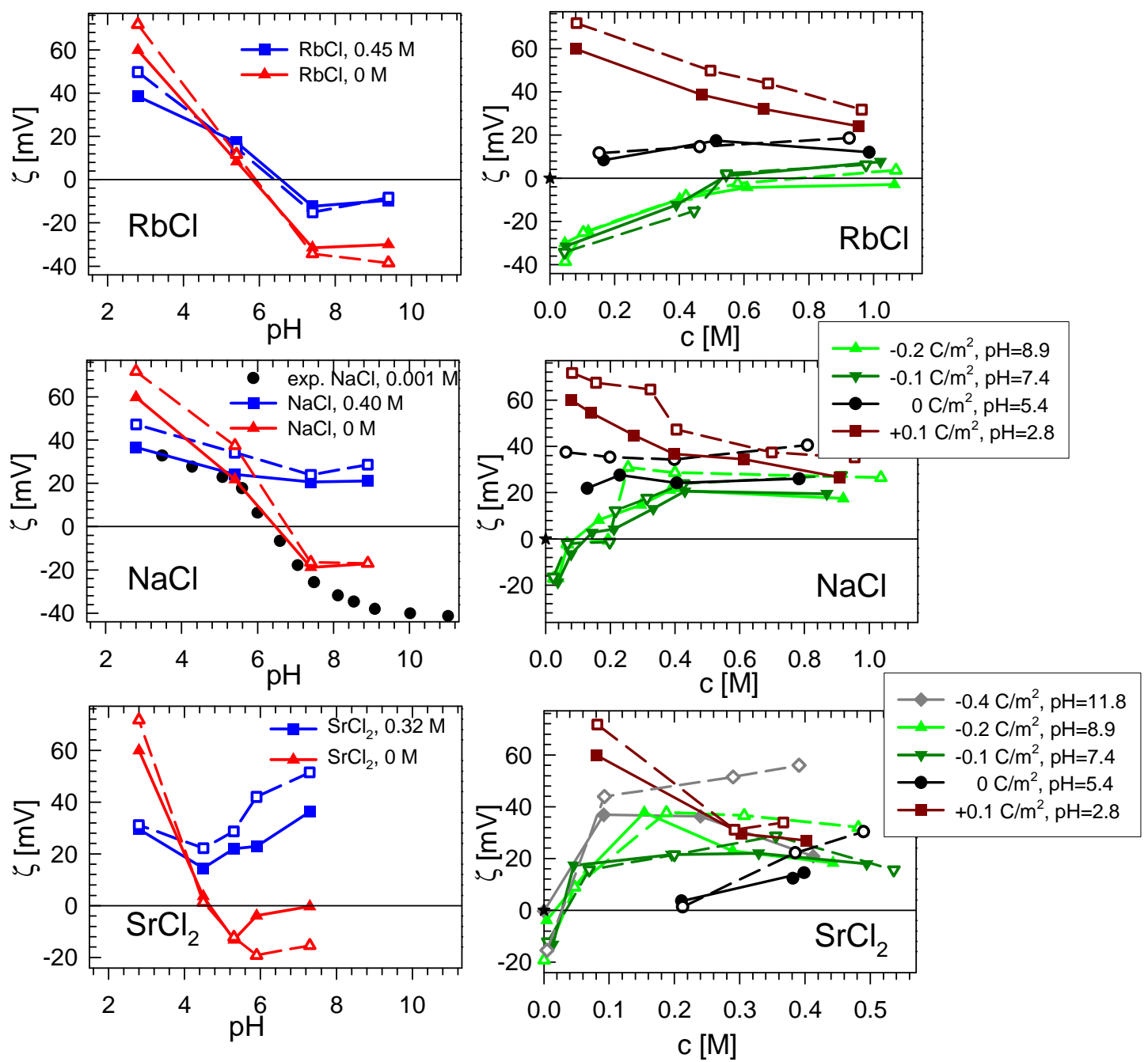

Figure SI9: ZPs $(\zeta)$ from NEMD (solid lines, filled symbols) and predicted from EMD (dashed lines, open symbols). $\mathrm{pH}$ dependence for higher and lowest bulk concentrations of ions (left) and concentration dependence (right). Reference experimental data are given as black symbols (left). 

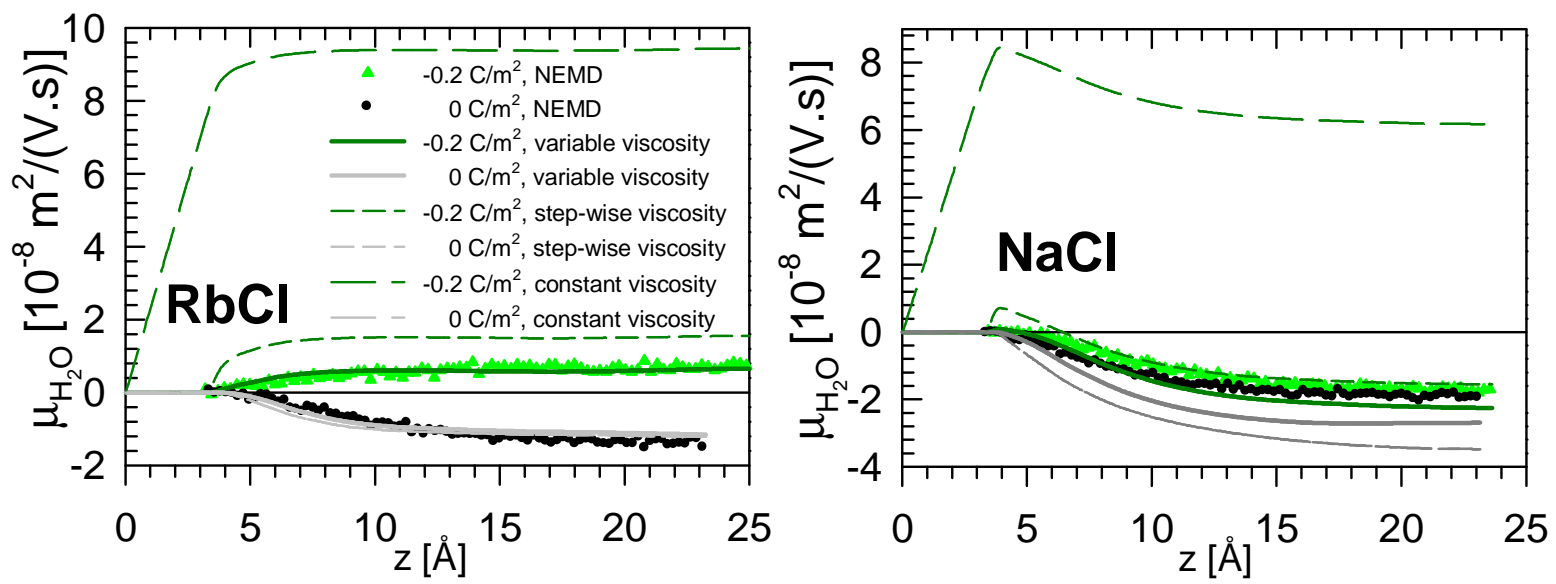

Figure SI10: Electroosmotic mobilities of water in $0.44 \mathrm{M} \mathrm{RbCl}$ (left) and $0.41 \mathrm{M} \mathrm{NaCl}$ (right) solutions from NEMD (points), EMD using viscosity profile from Figure SI7 (variable viscosity, solid lines), EMD using bulk viscosity $\left(8.9 \times 10^{-4} \mathrm{~Pa}\right.$.s) for distances larger than $3.4 \AA$ and infinite for smaller distances (step-wise viscosity, short dashed lines), and EMD using bulk viscosity $\left(8.9 \times 10^{-4} \mathrm{~Pa} . \mathrm{s}\right)$ for all distances (constant viscosity, long dashed lines).

\section{REFERENCES}

(1) Předota, M.; Machesky, M. L.; Wesolowski, D. J.; Cummings, P. T. Electric Double Layer at the Rutile (110) Surface. 4. Effect of Temperature and $\mathrm{pH}$ on the Adsorption and Dynamics of Ions. J. Phys. Chem. C 2013, 117, 22852-22866.

(2) CRC Handbook of Chemistry and Physics; $89^{\text {th }}$ ed., 2009.

(3) Palmer, B. J.; Pfund, D. M.; Fulton, J. L. Direct Modeling of EXAFS Spectra from Molecular Dynamics Simulations. J. Phys. Chem. 1996, 100, 13393-13398.

(4) Lee, S. H.; Rasaiah, J. C. Molecular Dynamics Simulation of Ion Mobility. 2. Alkali Metal and Halide Ions Using the SPC/E Model for Water at $25^{\circ} \mathrm{C}$. J. Phys. Chem. 1996, 100, 1420-1425.

(5) Berendsen, H. J. C.; Grigera, J. R.; Straatsma, T. P. The missing term in effective pair potentials. J. Phys. Chem. 1987, 91, 6269-6271.

(6) Delgado, A. V.; Gonzalez-Caballero, E.; Hunter, R. J.; Koopal, L. K.; Lyklema, J. Measurement and interpretation of electrokinetic phenomena - (IUPAC technical report). Pure Appl. Chem. 2005, 77, 17531805 .

(7) Qiao, R.; Aluru, N. R. Charge Inversion and Flow Reversal in a Nanochannel Electro-osmotic Flow. Phys. Rev. Lett. 2004, 92, 198301.

(8) Předota, M.; Bandura, A. V.; Cummings, P. T.; Kubicki, J. D.; Wesolowski, D. J.; Chialvo, A. A.; Machesky, M. L. Electric Double Layer at the Rutile (110) Surface. 1. Structure of Surfaces and Interfacial Water from Molecular Dynamics by Use of ab Initio Potentials. J. Phys. Chem. B 2004, 108, 12049-1206o.

(9) Předota, M.; Cummings, P. T.; Wesolowski, D. J. Electric Double Layer at the Rutile (110) Surface. 3. Inhomogeneous Viscosity and Diffusivity Measurement by Computer Simulations. J. Phys. Chem. C 2oo7, 111, 3071-3079.

(10) Parez, S.; Predota, M. Determination of the distance-dependent viscosity of mixtures in parallel slabs using non-equilibrium molecular dynamics. Phys. Chem. Chem. Phys. 2012, 14, 3640-365o. 